\title{
INCLUSIÓN SOCIAL DE JÓVENES CON DISCAPACIDAD COGNITIVA LEVE EN EL ÁMBITO EDUCATIVO
}

Social Inclusión of Young People with Mild Cognitive Disability in the Educational Ambit

\author{
Ana María Sastoque Uribe \\ anasastoque8991@hotmail.com \\ María Elena Lanza Martínez \\ mlanza@unicolmayor.edu.co \\ Universidad Colegio Mayor de Cundinamarca \\ Trabajo social Semillero Epistemes, Comprensión y Acción \\ Colombia
}

\begin{abstract}
Resumen
La presente memoria es producto de la investigación "Interacciones sociales en la inclusión social y educativa de estudiantes con discapacidad cognitiva leve en la institución educativa Villemar El Carmen IED” que pretende analizar las interacciones sociales existentes entre estudiantes con discapacidad cognitiva leve y sus compañeros del grado sexto en los procesos de inclusión social - educativa del Colegio Villemar El Carmen IED, desde el enfoque mixto, constituido por un alcance de nivel de investigación exploratorio y mediante el Diseño Transformativo Secuencial (DITRAS), tomando como perspectiva teórica el Construccionismo Social de Berger y Luckmann (1998). Dentro de la metodología, como técnicas de recolección de la información se empleó (desde la visión cuantitativa) el sociograma, y el grupo focal (respondiendo a la visión cualitativa). Los principales resultados permiten evidenciar que las interacciones sociales que se gestan entre estudiantes con y sin discapacidad cognitiva dentro del contexto de inclusión social y educativa se caracterizan en gran medida por agresiones directas e indirectas como amenazas y burlas, distanciamiento social, indiferencia, rechazo y superioridad. Y están enmarcadas en el discurso hegemónico de la discapacidad. Desde la profesión es necesario trabajar en las cuestiones de inclusión de personas con discapacidad, con el propósito de garantizar su plena participación en los espacios académicos.
\end{abstract}

Palabras clave:

Interacciones sociales, discapacidad, inclusión social, inclusión educativa. 


\section{INTRODUCCIÓN}

La discapacidad es un concepto que ha cambiado de significado durante los años. Anteriormente era desconocido. Aquella persona con alguna condición diferencial morfológica o cognitiva era víctima de la exclusión, segregación o hasta exterminio. Sin embargo, con el avance tecnológico esta visión logró tomar un rumbo distinto, las condiciones que generaban la discapacidad eran netamente médicas, esta mirada ayudó a ampliar los procesos de segregación y estigmatización de las Personas Con Discapacidad (PCD), en diferentes ámbitos, específicamente en el educativo, con educación especial que pretendió en un principio reconocer el aprendizaje de las personas con discapacidad cognitiva y su derecho a la educación. No obstante, fue otra estrategia de división. En consecuencia, surge en el modelo social, que significo un cambio en la visión de educación, apareciendo el término Inclusión y afirmando la necesidad de generar una modificación en las estructuras sociales, en los entornos académicos la importancia de ir más allá de lo pedagógico, vinculando las interacciones sociales dentro del proceso.

\section{Estado del arte y justificación}

El abordaje teórico y de conocimiento hacia la discapacidad ha estado caracterizado en su historia por la condición de salud-enfermedad otorgada por el modelo médico-rehabilitador desde el enfoque clínico. Hasta la llegada del modelo social, en los años 80's en Norteamérica y países europeos, se motivó a desarrollar estudios desde la rama de conocimiento de las ciencias sociales; no fue hasta hace poco que esta iniciativa comenzó a presentarse en países latinoamericanos, sin embargo por su reciente llegada, los estudios relacionados son limitados, entre ellos en el ámbito internacional se puede destacar la tesis doctoral denominada "Actitudes de estudiantes sin discapacidad hacia la inclusión de estudiantes con discapacidad en la educación superior" de Marcela Salinas quien hace una mirada en las actitudes de estudiantes sin discapacidad hacia los estudiantes con discapacidad y las variables que se le atribuyen a estas formas de relacionamiento para la inclusión de las PCD en la educación superior (Salinas, M., 2014); y la investigación desarrollada por Bautista, M., Turnbull, B., Saad, E. y Vidal, A., nombrada "La interacción de alumnos con discapacidad y sus pares en la escuela regular" cuyo objetivo se orientó a identificar las formas de interacción dadas en dos escuelas regulares de alumnos con discapacidad cognitiva y sus pares, como resultado halló 4 diferentes formas de relaciones (hostilidad, indiferencia, condescendencia y amistad), evidenciando la importancia de los factores psicosociales en los procesos de inclusión (Bautista, M., Turnbull, B., Saad, E. y Vidal, A., 2016); en el ámbito nacional los estudios obtenidos frente este eje temático se destaca "La atención educativa de niños, niñas y jóvenes considerados con necesidades especiales: Una mirada desde la integración y desde la inclusión" de Norelly Soto Builes quien construyó una teoría sobre los significados que subyacen a las comprensiones que existen sobre el proceso de atención educativa de niños, niñas y jóvenes considerados con necesidades educativas (Soto, N., 2009), cabe precisar que los trabajos desarrollados en trabajo social es poco frecuente el tema de la discapacidad, y pocos han desarrollado el tema de las relaciones sociales en la inclusión, además, los estudios gestados han pretendido desarrollar una mirada tradicionalista del modelo médico - rehabilitador, desde lo cual la profesión se enmarca en los ámbitos de asistencia. Sin embargo, con el modelo, la mirada se transforma, y se propone una construcción social que responda a una inclusión que defienda los derechos de las PCD (fig. 1), motivo por el cual se gesta esta iniciativa.

Figura 1. Integración debates conceptuales del Trabajo Social y la Discapacidad

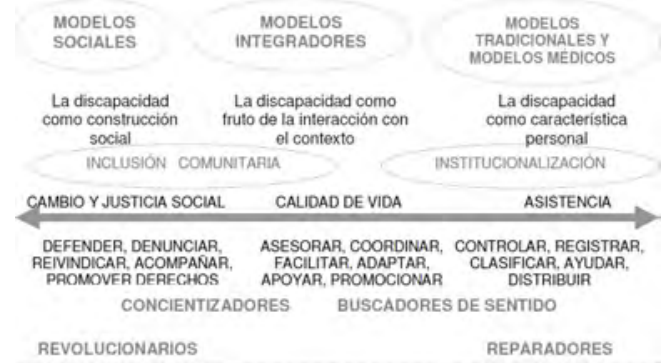

Recuperado de: Trabajo social y discapacidad intelectual en centros residenciales y de día en Andalucía. Una aproximación desde la teoría fundamentada. Díaz Jiménez., 2011. Doctoral dissertation, Universidad Pablo de Olavide.

Planteamiento del problema 
La inclusión desde la parte interaccional a nivel nacional se considera un reto, ya que los actores partícipes del ambiente escolar traen consigo representaciones y percepciones de las PCD otorgadas por los mismos entes hegemonices que producen su conocimiento y propician el alejamiento o cercanía con esta población (Bautista, Turnbull, Saad y Vidal, 2016). En concordancia la investigación desarrollada por Bautista, Turnbull, Saad y Vidal denominada "La interacción de alumnos con discapacidad y sus pares en la escuela regular" se encontró que las interacciones de los PCD en el ambiente escolar se propiciaban principalmente: las interacciones de hostilidad, indiferencia, condescendencia y amistad.

Estas formas de interacción de hostilidad, indiferencia y condescendencia generan en los individuos la incapacidad de establecer una interacción mutua, mirada desde iguales, en ocasiones caracterizada por formas de violencia directa e indirecta. Dichas interacciones se producen por la creencia de temor a lo diverso, y como consecuencia el rechazo y menosprecio (Bautista, Turnbull, Saad y Vidal, 2016). Las interacciones que se generan entre PCD y compañeros, profesores, y demás miembros participantes del ámbito escolar, se caracterizan en algunos casos por la discriminación, estigmatización, exclusión social en el mismo proceso educativo de las PCD que se promueve en las mismas aulas de clase.

Al no darse de forma adecuada las interacciones sociales de las PCD con los demás estudiantes, se provoca una falla en el proceso de inclusión social y educativo que puede provocar acciones o situaciones de exclusión generando deficiencias en la garantía de algunos derechos fundamentales de dicha población que se encuentran consignados en la Constitución Política de Colombia y en la Convención de Derechos de las Personas con Discapacidad, como la educación.

\section{Tópicos teóricos}

Se toman diferentes referentes conceptuales como: discapacidad, discapacidad cognitiva, inclusión social de PCD, inclusión educativa de PCD, interacciones sociales, tipos de interacción, posicionamientos sociales, que serán expuestos a continuación brevemente:

Discapacidad: La producción del conocimiento de la discapacidad, ha estado envuelta por las mentes individuales hegemónicas que conforman un grupo social, con el propósito de abordar otras realidades desde su cultura occidental llamada "realidad absoluta" (Gergen, 1997, citado por López, 2013). En concordancia Gergen, (1996) citando a Foucault (1980), argumenta que el saber está relacionado con el poder, debido a que las estructuras del poder influyen en los enclaves culturales permitiendo que algunas poblaciones sean mayormente favorecidas que otras, por lo cual se establecen jerarquías de privilegios y se fomenta la activación de polaridades, la institucionalización de esta influencia en los enclaves culturales conlleva a procesos de exclusión. (Foucault, 1980, citado por Gergen, 1996).

En este sentido, las teorías de la discapacidad que le otorgan la connotación de déficit como ideología borran los procesos sociales por los cuales ha transcurrido. Desde esta perspectiva, Rosato y sus colaboradores (2009) mencionan que en el campo de la discapacidad se han instaurado políticas de alteridad pensadas desde la visión hegemónica, que han operado en discursos científicos, prácticas profesionales e institucionales generando una comprensión de esta. Estas políticas han otorgado una delineación conceptual biologicista que produce desventajas con relación a los "normales" -quienes no conllevan esta connotación de déficit- (Rosato et al, 2009), en este sentido la OMS, dentro de su Clasificación Internacional del Funcionamiento, la Discapacidad y la Salud (CIF), instaura esta visión occidental al legitimar la discapacidad desde tres elementos: Las deficiencias encaminadas al problema con las estructuras o funciones corporales, las limitaciones de la actividad, referidas a las dificultades que una persona puede tener en la realización de sus actividades vitales y por último la restricción de la participación que son los problemas que una PCD puede experimentar al involucrarse con otras personas de su entorno (OMS, 2001).

Es decir, en tanto las políticas específicas sigan estando sostenidas y legitimadas por modos hegemónicos de entender a la discapacidad como fenómeno del orden de lo biológico, como tragedia no elegida que le ocurre a algunos sujetos, y por lo tanto, como cuestión individual, continuarán siendo modos de producción de discapacidad y discapacitados reforzando por efecto de ideología de la 
normalidad, lo que Oliver (2008:27) denomina «burocracia de la asistencia social en discapacidad» (Oliver, 2008, p.27 citado por Rosato et al, 2009, p. 103)

Como una mirada en contraposición a la postura hegemónica de la discapacidad (otorgada por la OMS) Puga en 2004, denota que:

El término discapacidad no es inherente a la persona, no está determinado exclusivamente por factores biomédicos. El etiquetado de discapacidad como fenómeno médico ignora la complejidad de factores que (sumados a los médicos) conducen a una limitación para desarrollar actividades sociales, a una desventaja social (Puga, 2004. p. 12).

Discapacidad Cognitiva: Según el Instituto Colombiano de Bienestar Familiar (ICBF) en 2010, menciona que la discapacidad cognitiva está constituida por tres elementos centrales. El primero de ellos, es el entorno o contexto, que refiere a los lugares donde la persona habita; el segundo, son las capacidades, concebidas como los atributos del sujeto que permiten su funcionamiento adecuado en la sociedad; y el funcionamiento, que relaciona las capacidades con el entorno. Por ende, se afirma que si en el entorno se gesta una falla puede involucrar una barrera para el desarrollo de la persona (ICBF, 2010).

Inclusión social: Según la Ley 1618 de 2013, define la inclusión desde una visión social como:

Proceso que asegura que todas las personas tengan las mismas oportunidades, y la posibilidad real y efectiva de acceder, participar, relacionarse y disfrutar de un bien, servicio o ambiente, junto con los demás ciudadanos, sin ninguna limitación o restricción por motivo de discapacidad, mediante acciones concretas que ayuden a mejorar la calidad de vida de las personas con discapacidad (Ley 1618, 2013, p. 2).

Sin embargo, la inclusión social es un término ideal. Pensar en una persona socialmente incluida permite considerar lo contrario, la exclusión social a la cual Ramírez íñiguez en 2016 refiere como:

Expulsión o restricción de los sujetos para acceder a bienes fundamentales como la educación, la vivienda, el trabajo o la salud, los cuales son considerados derechos básicos para el desarrollo de cualquier persona (Benito, 2010; Osler y Starkey, 2005; Ritacco y Luengo, 2012 citado por Ramírez Íñiguez, 2016, p. 179).

Es decir, la inclusión social alude a las posibilidades del sujeto para acceder a las necesidades de desarrollo social y personal como acceso de bienes: educación, vivienda, trabajo y salud. Según Ramírez Î́niguez en 2016, una de las bases para lograr la inclusión social es la educación, que permite desarrollar competencias para afrontar la sociedad y participar en ella, así como lo plantean también Echeita y Simón en 2007, que expresan:

La educación escolar es hoy la principal garantía del derecho de todos a aprender (Darling-Hamond, 2001 citado por Echeita y Simón, 2007) y, por lo tanto, la llave más importante para la puerta de entrada a la inclusión social (Echeita y Simón, 2007, p. 2).

Inclusión educativa e interacciones sociales: Bautista, Turnbull, Saad y Vidal, 2016, la inclusión de las PCD en la educación no es un aspecto netamente pedagógico, sino que también influyen diversos actores externos a este tales como: las interacciones sociales, el aprendizaje de roles, la aceptación de valores, etc.

Desde la postura construccionista, Perdomo (2002) refiere que las interacciones entre los sujetos en la cotidianidad generan significaciones mediante el lenguaje. En consecuencia, el discurso que se da en determinada comunidad se va construyendo, resignificando y transformando en el marco de las interacciones, es decir, por medio de las relaciones iniciamos la reconstrucción del mundo (Perdomo, 2002). En tal sentido, las relaciones entre sujetos constituyen verdades, que forman una realidad, esta verdad es cuestión de perspectivas (producto de intercambios sociales y de acuerdo), los sujetos son "quienes generan las distinciones que definen como "realidad" a partir de su propia realidad" (Donoso, T., 2004, p, 11)

En congruencia, las interacciones sociales desde Arias (2009) son las vivencias cotidianas que se propician dentro de las aulas de clase. Por ende, las relaciones que se gestan están empapadas de 
opciones valorativas que se visualizan por medio de la interacción comunicativa, las acciones, actitudes y comportamientos.

En cuanto a Tünnermann (2011), menciona que "la interacción social favorece el aprendizaje por medio de la socialización entre alumnos permitiendo la construcción de un ambiente de aprendizaje constructivista y motivacional que permite crear objetivos y metas comunes" (Tünnermann, 2011, citado por Álvarez y Cázares, 2018, P.12). En este sentido, las interacciones sociales se desarrollan dentro de los procesos de socialización, donde el individuo comienza a hacer parte de una sociedad.

Dentro de dicha socialización, Berger y Luckman (1986) mencionan que se gestan situaciones de carga emocional que permite el aprendizaje del sujeto en relación con los otros significantes, mediante el proceso de internalización e identificación desde los roles y actitudes de los otros, el sujeto crea su propia identidad y se considera "una entidad reflejada, [...] llega ser lo que los otros significantes lo consideran" (P.165). El sujeto al conocer el mundo de los otros, remite parte de esas experiencias a su propia identidad y muestra parte de la cultura e ideas que se encuentran inmersas durante su socialización primaria.

Posicionamientos sociales: Estévez, Martínez, y Jiménez (2009) mencionan que las relaciones sociales en la escuela se originan a partir de agrupaciones formales e informales que contienen normas establecidas en cada una. Las relaciones y agrupamientos se conforman de acuerdo con las metas y normas inherentes a la cultura a la que corresponden los adolescentes, e igualmente a las normas estipuladas en cada grupo que los diferencian de otros. En cada uno de ellos existen diferentes relaciones de poder que concuerdan a un posicionamiento social, los integrantes mayormente aceptados ocupan las partes centrales del grupo, mientras que los menos aceptados ocupan los sitios externos.

En este sentido Coie, Dodge y Kupersmidt, 1990; Gifford-Smith y Brownell, 2003; Hill y Merrell, 2009; Newcomb, Bukowski, y Pattee, 1993 citados por Estévez, Martínez y Jiménez 1990, mencionan los posicionamientos sociales que se evidencian en las aulas académicas que son los siguientes:

Popular: son aquellos adolescentes que son aceptados por la mayoría de sus compañeros. El calificativo de popularidad se atribuye al prestigio (por actividades académicas) aspectos emocionales, o por manifestar los atributos ideales del grupo. Rechazado: refiere a los adolescentes que no generan empatía con el resto de sus compañeros, contempla conductas agresivas, disruptivas y al quebrantamiento de las normas institucionales, tienen relaciones conflictivas entre sus compañeros y docentes, presentan baja competencia social y académica. También se caracterizan por tener conductas retraídas, depresión y ansiedad. Ignorado: son aquellos adolescentes que resultan ser invisibles para sus demás compañeros, reciben poca atención, usualmente son tímidos, pacíficos y reservados, respetan las reglas, son sujetos comprometidos en las actividades socialmente aceptadas. Promedio: son adolescentes que no se destacan, no corresponden a los ignorados pues son mayormente visibles. Controvertido: son adolescentes que tienen iguales niveles de aprobación o rechazo, les agradan a muchos, pero a otros no. Contemplan comportamientos prosociales y antisociales.

Tipos de interacción: los posicionamientos sociales se atribuyen a las distintas interacciones que se gestan al interior de la escuela entre pares académicos (estudiantes con y sin discapacidad) según Bautista, Turnbull, Saad y Vidal, 2016 entre las que se encuentran:

Interacciones de hostilidad: las relaciones que se dan se caracterizan por distintos grados de agresividad (directa o indirecta) producido por uno o unos hacia otro(s). Interacciones de indiferencia: la relación es indirecta, coexisten en un mismo lugar, sin embargo, no interactúan entre sí. Interacciones de condescendencia: es el tipo de relación en donde el individuo se posiciona en un rango mayor que el otro dentro de la interacción, y lo ve inferior, se percibe como un sujeto extraño y no se logra una interdependencia entre iguales. No existe un compromiso de vinculación mayor. Interacciones de amistad: estas relaciones promueven un alto grado de reciprocidad, generando compañerismo, fraternidad, empatía profunda, compromiso y correspondencia. 


\section{Método}

A continuación, se expone el diseño metodológico de la investigación, en el que se explica lugar, población, autor metodológico, La investigación se desarrolló en la Institución Educativa Distrital (IED) Villemar el Carmen, a la cual asisten niños, niñas y adolescentes provenientes de la localidad de Fontibón, Ciudad de Bogotá D.C., Colombia. Dicho colegio, cuenta con profesores de apoyo para el proceso de inclusión educativa de estudiantes con discapacidad cognitiva leve y moderada; y educación especial a estudiantes con discapacidad cognitiva más profunda.

En cuanto a la población, se abordó a todos los estudiantes con discapacidad cognitiva del frado sexto, y siete de sus compañeros elegidos de forma aleatoria.

Dentro del diseño de la investigación, se eligió autor metodológico a Hernández, (2014). La investigación se propició desde el enfoque mixto por medio del Diseño Transformativo Secuencial (DITRAS), orientado por la perspectiva teórica del construccionismo social. Se empleó el método cualitativo para la comprensión de los estudiantes hacia la discapacidad y las interacciones entre compañeros con discapacidad cognitiva leve y sus pares académicos. Con el método cuantitativo se pretendió categorizar, medir y analizar las preferencias relacionales de los estudiantes con discapacidad cognitiva y sus compañeros en los procesos de inclusión social educativa.

La perspectiva teórica de la investigación es el construccionismo social, que posibilita la comprensión de las subjetividades de los estudiantes de sexto grado regulares frente a sus compañeros con discapacidad, además se basa en las relaciones sociales surgidas de las intersubjetividades y de las construcciones sociales de conocimiento desde sus propios actores participantes, en este caso, los estudiantes de sexto grado con discapacidad y sus compañeros dentro del colegio Villemar, el Carmen IED.

El alcance de la investigación es exploratorio, dado que el campo de la discapacidad ha sido estudiado por disciplinas relacionadas con lo médico, otorgando un vacío en el entendimiento de esta desde el ámbito social, cuya mirada se propició en los años 2000, generando una apertura investigativa en el campo de la discapacidad.

Para el desarrollo de la investigación, se emplean las siguientes fases:

Fase preparatoria: en esta etapa se desarrolló el diseño de la investigación.

Fase de trabajo de campo y de recolección de información: en este periodo, se realizó la aproximación a la institución educativa, se selecciona la muestra y se procede a aplicar los instrumentos, sistematizar la información y discriminar. En este sentido, Las técnicas e instrumentos de recolección de información responden al método mixto, que a continuación, se expondrán de forma detallada:

\begin{tabular}{|c|c|c|}
\hline Técnicas & Instrumentos & Definición y Justificación \\
\hline Sociograma & Test sociométrico & $\begin{array}{l}\text { El sociograma es una técnica que permite estudiar las preferencias relacionales que coexisten dentro de un grupo de personas, representa } \\
\text { su estructura y permite la obtención de una radiografía grupal (Bautista et al., 2009). Se compone de un test sociométrico que consiste en } \\
\text { preguntar a los integrantes del grupo con quienes desearían trabajar y con quienes no; y de un diagrama que permite observar las relaciones } \\
\text { de cada miembro con lo demás, permitiendo la identificación de los posicionamientos de los estudiantes con discapacidad cognitiva dentro } \\
\text { del aula académica. } \\
\text { Este instrumento se aplicó a todos los estudiantes de los cuatro cursos de grado sexto. Se identificaron los estudiantes con discapacidad } \\
\text { cognitiva de acuerdo con los votos de aceptación y de rechazo hacia estos, clasificándolos en popular, ignorado, controvertido, promedio } \\
\text { y rechazado; estos se diferencian de acuerdo a las cantidades de votos de aceptación y rechazo. Por ello se considera a Coie, Dodge y } \\
\text { Kupersmidt, 1990; Gifford-Smith y Brownell, 2003; Hill y Merrell, 2004; Newcomb, Bukowski, y Pattee, } 1993 \text { citados por Estévez, Martínez } \\
\text { y Jiménez (2009) mediante la siguiente escala: } \\
\text { Popular: Mayor número de aceptación en el total general. } \\
\text { lgnorado: Pocos votos de aceptación y pocos de rechazo, pero en cantidad lo aceptan más o viseversa. } \\
\text { Controvertido: El total general es 0, es decir lo rechazan y aceptan la misma cantidad de votos. } \\
\text { Promedio: Número alto de aceptación, pero menores a la del líder o número de rechazo, pero menores al del rechazado. } \\
\text { Rechazado: Mayor número de rechazo. } \\
\text { Se emplean las siguientes instrucciones dentro del proceso: } \\
\text { Elige con qué } 3 \text { compañeros o compañeras de clase te gustaría estar en el mismo equipo Indica con qué } 3 \text { compañeros o compañeras de } \\
\text { clase preferirías que no estuviera en tu mismo equipo }\end{array}$ \\
\hline Grupo Focal & $\begin{array}{l}\text { Entrevista } \\
\text { semiestructurada }\end{array}$ & $\begin{array}{l}\text { Es una técnica cualitativa en la cual se dialoga sobre una temática específica, con preguntas ya establecidas en este caso sobre las personas } \\
\text { con discapacidad. Se realizan dos grupos: Estudiantes con discapacidad cognitiva y siete de sus compañeros. } \\
\text { Esta técnica tiene como finalidad conocer las percepciones de los estudiantes acerca de las PCD y averiguar los significados que tienen } \\
\text { relacionados con la discapacidad. }\end{array}$ \\
\hline
\end{tabular}


Para la realización de la investigación se contactó con la IED, iniciando la recolección de la información en el segundo semestre del año 2019, a partir del mes de septiembre hasta la primera semana del mes de noviembre. Se retomó el estudio en las dos primeras semanas del mes de febrero del 2020, aplicando los instrumentos de recolección de información los martes en la Jornada de la mañana.

Fase analítica: se toma en cuenta la información y los datos recolectados y según Hernández (2014), se procede a "analizar los datos; en los métodos mixtos, el investigador confía en los procedimientos estandarizados y cuantitativos (estadística descriptiva e inferencial), así como en los cualitativos (codificación y evaluación temática), además de análisis combinados" (p. 574).

Para el análisis de la información, se consideró el tipo de investigación mixto, por lo tanto, se aplicaron y analizaron instrumentos cuantitativos y cualitativos, los procesos se describen a continuación:

Desde el método cuantitativo se consideraron los votos de aceptación y de rechazo hacia los estudiantes con discapacidad cognitiva, empleando el siguiente proceso:

Se tabulo y construyó una matriz de Excel de los cuatro cursos del grado sexto Jornada mañana de la IED, en la cual se colocaron inicialmente todos los nombres de los estudiantes de forma vertical y horizontal.

Posteriormente se ubicaron los nombres cada uno de los estudiantes de forma vertical para poder denotar a los estudiantes que aceptan y rechazan en la parte horizontal de la matriz.

Considerando los objetivos de la investigación, se tomaron en cuenta a los estudiantes que tenían relación con los estudiantes con discapacidad y se graficaron con el uso de triángulos y círculos, los cuales simbolizaban de forma correspondiente hombre y mujer. Además, se utilizaron líneas de colores para marcar la aceptación (azules) y para el rechazo (verdes).

A partir de la matriz y de los gráficos obtenidos se clasificó a cada uno de los estudiantes con discapacidad cognitiva leve en uno de los posicionamientos sociales descritos en la Tabla 1.

Desde el método cualitativo se siguieron los siguientes pasos:

Transcripción de los grupos focales.

Lectura y comparación entre los grupos focales.

Generación de categorías y subcategorías de los datos cualitativos a partir de las respuestas obtenidas en los grupos focales y las justificaciones de la aceptación y rechazo hacia los estudiantes con discapacidad cognitiva leve en el sociograma.

Análisis y obtención de los resultados relacionados con la discapacidad, discapacidad cognitiva, y las interacciones entre los estudiantes.

\section{Resultados}

A partir de la aplicación de las técnicas de entrevista y de sociograma por medio de sus respectivos instrumentos, se obtienen los siguientes resultados:

\section{Posicionamientos sociales}

Desde la parte cuantitativa se empleó el sociograma que tiene como finalidad observar por medio de las elecciones de rechazo o de aceptación las posiciones sociales en las cuales se ubican los estudiantes con discapacidad cognitiva respecto a sus demás compañeros de los cursos de sexto grado. Se aplicó a los 133 estudiantes que conforman al grado sexto, el $53 \%$ (71) son de género masculino y el $47 \%$ (62) son de género femenino como lo muestra la figura 2. En cuanto al total de los estudiantes de grado sexto el $95 \%$ de los mismos no tienen discapacidad cognitiva, mientras el $5 \%$ cuentan con este tipo de discapacidad (fig. 3) 


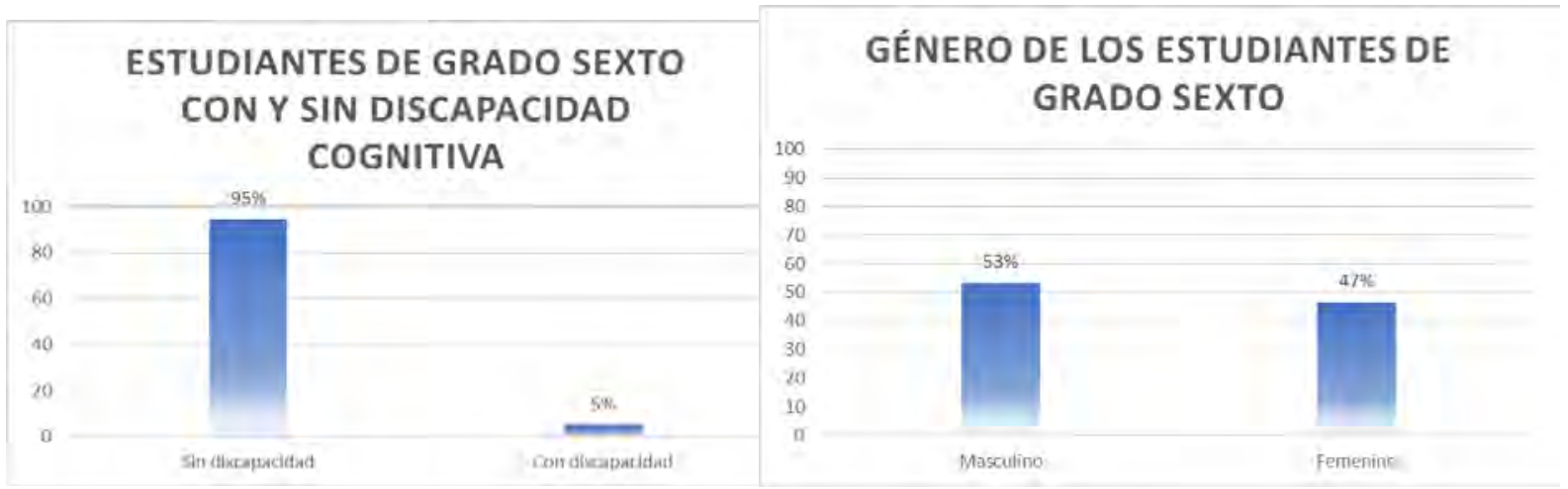

Figura 2 Y 3 Porcentaje de estudiantes con y sin discapacidad cognitiva del grado sexto

A continuación, se presentan los datos de los estudiantes con discapacidad cognitiva leve y las posiciones sociales en las que se encuentran de acuerdo con lo manifestado por sus compañeros:

\begin{tabular}{|l|llll|}
\hline$\#$ & Curso & Aceptación & Rechazo & Promedio \\
\hline 33 & 601 & 0 & 15 & Rechazado \\
1 & 602 & 4 & 3 & Promedio \\
35 & 602 & 1 & 2 & Ignorado \\
2 & 603 & 0 & 26 & Rechazado \\
19 & 603 & 3 & 1 & Ignorado \\
38 & 603 & 2 & 0 & Ignorado \\
24 & 604 & 4 & 10 & Promedio \\
\hline
\end{tabular}

Tabla 2. Puntaje de aceptación, rechazo y posicionamiento de los estudiantes con discapacidad cognitiva leve. Elaboración propia

Cada uno de los posicionamientos corresponden a los puntajes obtenidos por cada uno de los estudiantes. Los posicionamientos de rechazo corresponden al mayor número de votos de rechazo obtenido, en este encontramos a los estudiantes \#33 del grado 601 (fig. 3) y el \#2 del curso 603 (fig. 4)
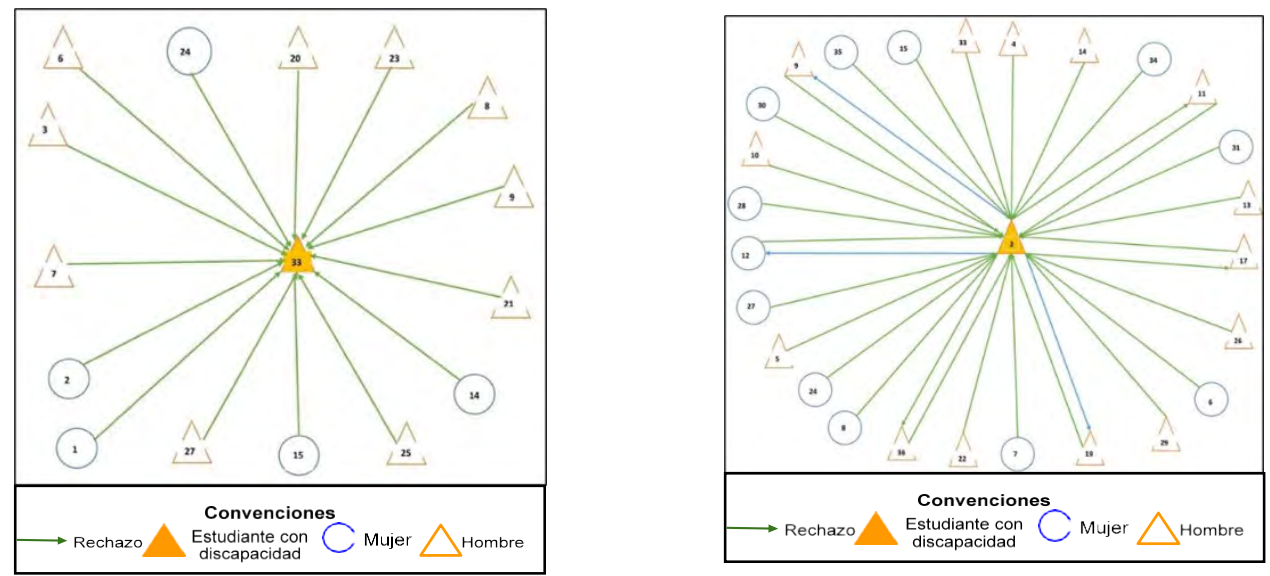

Figura 4 y 5 Diagrama de elecciones estudiante \#2. Elaboración propia

En el posicionamiento social de ignorado se ubican los estudiantes \#35 del grado 602 (fig. 5), el \#19 (fig. 6) y \#38 (fig. 7) del curso 603 que obtuvieron pocos botos de aceptación y de rechazo. 

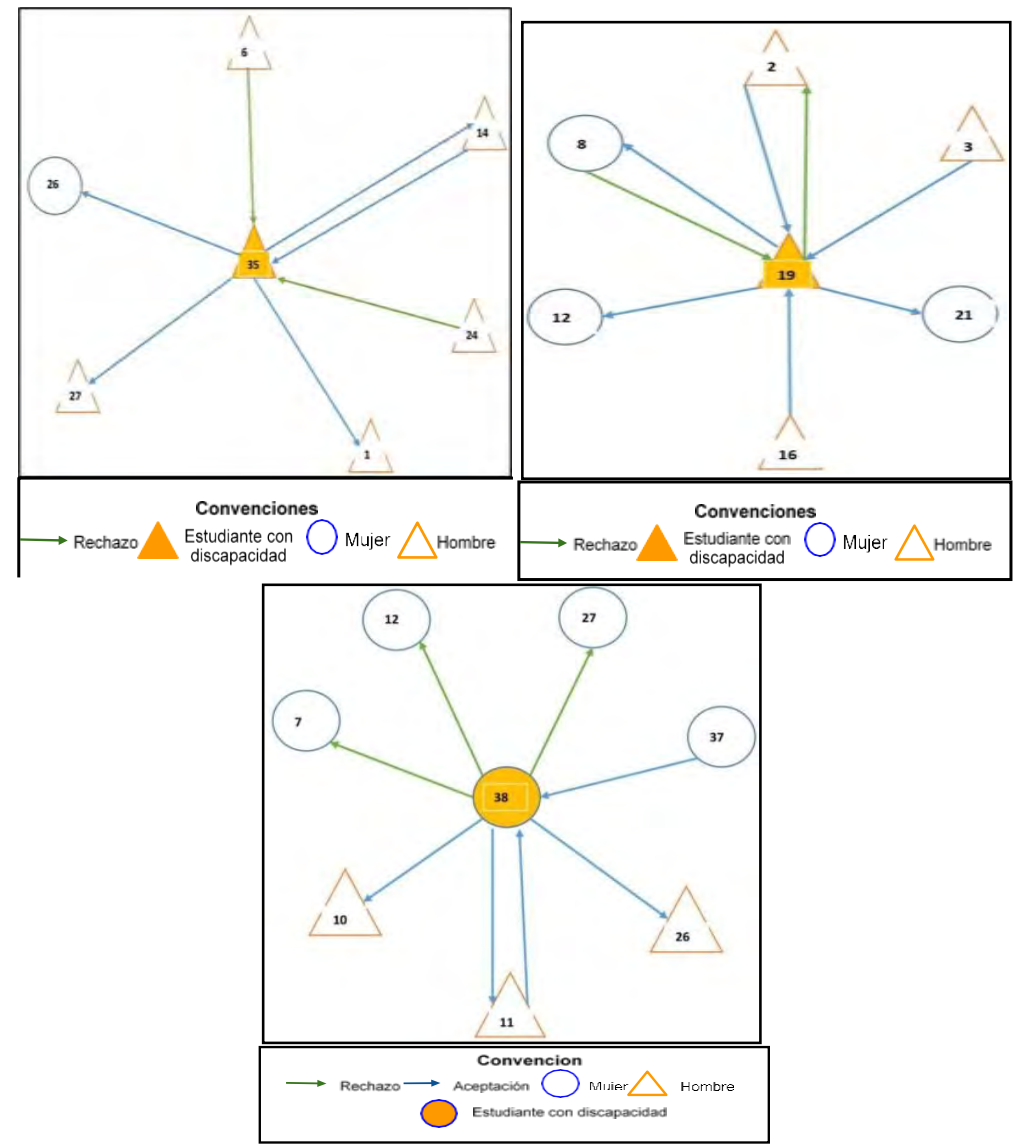

Figura 6, 7 y 8 Diagrama de elecciones

En cuanto al posicionamiento social de promedio, se ubican los estudiantes \#1 del curso 602 (fig. 8), y el \#24 del grado 604 (fig. 9), quienes contienen números de aceptación o rechazo altos, menores al rechazado o al líder.

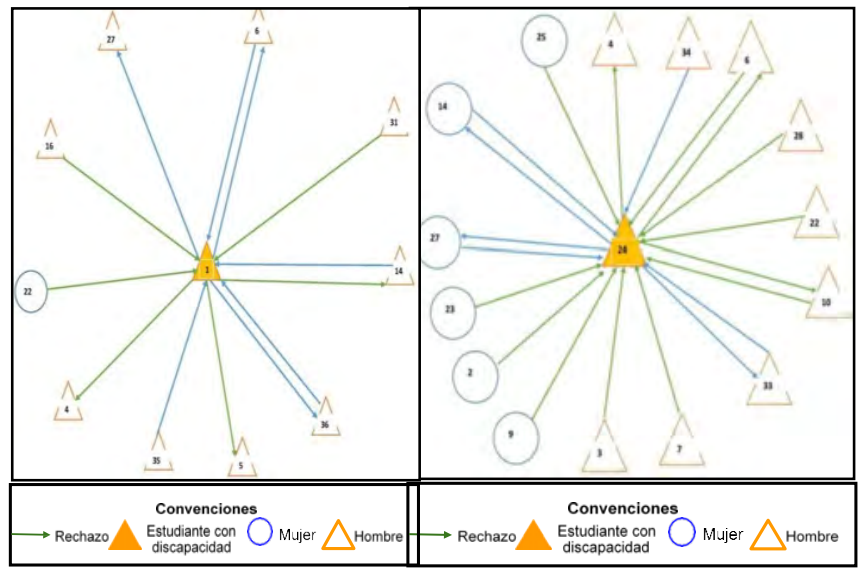

Figura 9 y 10 Diagrama de elecciones estudiante \#1. Elaboración propia

Dentro de la dinámica interna del grupo se manifiesta que las interacciones sociales dentro de los cursos de sexto se gestan a partir de una agrupación formal. En cada uno de ellos se evidencian relaciones de poder que corresponden a determinados posicionamientos sociales, en los cuales los estudiantes con discapacidad cognitiva leve ocupan los lugares periféricos, encontrándose en los posicionamientos de rechazado, promedio e ignorado. Las características por las cuales se sitúan en dichas posiciones son: poca visibilidad, desempeño académico, relaciones conflictivas, empatía y aspectos emocionales.

Imaginarios de la Discapacidad 
El conocimiento respecto a la discapacidad es la pretensión hegemónica de las mentes individuales que tienen el propósito de abordar otras realidades desde su realidad "absoluta" generando polaridades que conllevan a procesos de exclusión (Gergen, 1996). En este sentido las teorías otorgadas por la OMS y el ICBF se vinculan directamente con los discursos que otorgan los estudiantes del grado sexto, estos aluden a la discapacidad y discapacidad cognitiva significados encaminados al déficit como las deficiencias y limitaciones expresadas con los siguientes argumentos: "tienen limitaciones" (Estudiante \#37 del grado 603, 2020), "físicas o mentales" (Estudiante \#37 del grado 603, 2020), "no pueden hacer todas las capacidades que el cuerpo les ofrece" (Estudiante \#3 del grado 601, 2020), "restricciones en algunas de las actividades" (Estudiante \#29 del grado 601, 2020); capacidades y funcionamiento expresadas en las siguientes narraciones: "Que a la persona no se formó bien" (Estudiante \#10 del grado 601, 2020), "que no tienen el mismo aprendizaje, que se les dificulta comprender y aprender" (Estudiante \#3 del grado 601, 2020), "se les dificulta aprender, ya que como es de inclusión entonces hay que ayudarle muchísimo, es intentar ayudar a alguien que tampoco se deja ayudar" (Estudiante \#3 del grado 601, 2020. Rosato y sus colaboradores (2009), mencionan que estas teorías borran los procesos sociales por los cuales han transcurrido las PCD, estos discursos han generado la comprensión de la misma desde el enfoque biologicista, de tal forma que los productores de esta demarcación distancian por medio de su discurso a los estudiantes con discapacidad cognitiva leve de los "normales" (sus compañeros) "permitiendo la reproducción de las condiciones para que tal demarcación se sostenga y legitime" (Rosato et al, 2009, p.102). Por lo cual al referirse a la discapacidad establecen la idea del déficit de cada persona, refiriéndose a una condición individualizada que dificulta la visualización de las barreras sociales, desmeritando la necesidad de generar medidas desde lo social.

\section{Tipos de interacción}

Los tipos de interacción social reconocidos dentro de las aulas de sexto son: de hostilidad, indiferencia, condescendencia y amistad. A continuación, serán analizado a profundidad:

Interacción de hostilidad: Se define como aquellas formas de relación en donde se manifiesta agresividad directa e indirecta, se produce de un actor o varios a otro u otros, o en ambos sentidos. Este tipo de relación se manifiesta dentro del grado sexto, los estudiantes con discapacidad cognitiva leve exponen actos violentos expresados en agresiones verbales y amenazantes hacia ellos:), "Mmmm desde que mi amiga ..., desde que tuvo un novio... y ella empezó a amenazarme" (Estudiante \#19 del grado 603, 2020), "Pues en un día que me había tocado, nos dijo un profesor que que tenían que quién eran de apoyo, entonces, yo levanté la mano, y todos se empezaron a burlar, bueno y, quedaron como tontos frente al profesor" (Estudiante \#1 del grado 602, 2020), sin embargo sus compañeros, manifiestan que las agresiones son causada por los actores con discapacidad cognitiva hacia el resto de sus compañeros: "es un compañero que me molesta y golpea a otros" (Estudiante \#3 del grado 601, 2019), "él es muy grosero y sólo se expresa pegando" (Estudiante \#22 del grado 602, 2019), "es fastidioso y solo busca problema" (Estudiante \#7 del grado 603, 2019),

En este marco discursivo, en el construccionismo social, el lenguaje es esencial en la interacción entre los sujetos, convirtiéndose en el mundo significativo de estos y permitiéndoles construir su identidad. Lo anterior, se evidencia por medio miradas contrapuestas hacia la misma situación, que constituyen un tipo particular de sujeto que a partir de su relacionamiento con diferentes grupos sociales va creando valoraciones compartidas. Para lo cual Berger y Luckmann (1986) brindan una visión congruente al referir que "los procesos subjetivos de otro [...]

se vuelven significativos para mí. Eso no significa que yo comprenda adecuadamente al otro" (p. 163). Es decir, las comprensiones respecto a la interacción de hostilidad son vistas desde el mismo enfoque de agresiones directas, sin embargo, en las narraciones se evidencia que, por un lado, los estudiantes con discapacidad cognitiva refieren a las mismas como burlas y amenazas recibidas por parte de sus compañeros. Mientras que, sus pares utilizan los términos de golpes y groserías propiciados por los estudiantes con discapacidad cognitiva hacia ellos.

Interacción de indiferencia: Pretende describir las relaciones en donde los actores que coexisten en un mismo lugar no son observados socialmente, los otros mantienen una actitud de inexistencia hacia el sujeto. Este tipo de interacción se manifestó en el grado sexto, sin embargo, su entendimiento fue contrario, los estudiantes con discapacidad cognitiva, expresaron sentimientos de soledad: "No, yo me 
siento muy solo" (Estudiante \#2 del grado 603, 2020) "Como no hablo con nadie" (Estudiante \#2 del grado 603, 2020) "Porque aparte de que naaa, nadie quiere hablar haha, por eso casi no hablo con nadie, pues obviamente hablo, pero solamente para actividades, que sea algo para hablar hablar, no" (Estudiante \#2 del grado 603, 2020). Mientras los argumentos de sus compañeros aluden que: "no es que los separemos, es que ellos se separan, ósea automáticamente así ellos, digamos estamos haciendo trabajos en grupo y todos comienzan a hacer los grupos y ellos como se quedan solos y les dice uno como bueno venga acá al grupo, no y se queda solo" (Estudiante \#3 del grado 601, 2020), "no es que nosotros lo excluyamos, sino que es el mismo que se excluye" (Estudiante \#3 del grado 601, 2020)

Respecto a lo anteriormente narrado, Perdomo afirma que la configuración de la interacción corresponde al lenguaje, en este sentido se evidencia que existe correlación en cuanto se manifiesta que hay un interaccionamiento de indiferencia, que se vuelve significativo y compartido, sin embargo es comprendido de formas diferentes por parte de los actores del grado sexto, en cuanto a los estudiantes con discapacidad cognitiva refieren sentimientos de soledad por el distanciamiento de sus compañeros, mientras, que los estudiantes sin esta condición en sus narraciones denotan la separación propia de las PCD a la hora de conformar grupos de trabajo. Las valoraciones surgidas dentro de este tipo de interacción refieren al distanciamiento de los propios sujetos en los grupos de trabajo con discapacidad cognitiva que permite la reproducción de este tipo de interacción.

Interacción de condescendencia: Se define como aquellas relaciones donde el individuo se posiciona en un rango mayor que el otro dentro de la interacción, y lo ve inferior, se percibe como un sujeto extraño y no se logra una interdependencia entre iguales, en este sentido, los compañeros de los estudiantes con discapacidad cognitiva del grado sexto manifiestan relatos como: "no, pues, ósea cuando me tocaba trabajar con alguno de ellos, me molesta, porque pues es más lento el desarrollo del trabajo" (Estudiante \#21 del grado 603, 2020), "es una persona que necesita ayuda profesional con el cual no me gusta convivir" (Estudiante \#21 del grado 601, 2019), "me cae mal y por qué es raro" (Estudiante \#28 del grado 603, 2019).

Respecto lo mencionado anteriormente, Salazar (2009) menciona que los procesos conceptuales propuestos por la hegemonía sobre la discapacidad han determinado parámetros en la interacción hacia la población con discapacidad, en esta dirección Rincón afirma que quienes se identifican como "normales", evidencian estas deficiencias y las valoran y a partir de las mismas entablan una relación con lo "atípico" (Rincón, 2009), en este sentido Perdomo refiere que las formas de realidad proporcionadas por el nodo científico intervienen en la forma como los sujetos se conciben a sí mismos y a los otros (Perdomo, 2002), por lo cual al catalogar a los estudiantes con discapacidad valoraciones como "raro" y "lento" (marcadas en el déficit) hacen ver a esta población como anormal, estipulando este tipo de interacción delimitado por este discurso de la alteridad - normalidad, que posiciona en un lugar inferior al estudiante con discapacidad respecto a sus compañeros sin esta condición.

Interacción de amistad: Dentro de las interacciones de amistad, se encuentran las relaciones que se determinan por reciprocidad, compañerismo, fraternidad, empatía y correspondencia. Este tipo de interacción se manifestó en los estudiantes del grado sexto que manifestaron: "son mis amigos y con los que siempre me hago" (Estudiante \#38 del grado 603, 2019), "son buenas personas y hacen buen trabajo" (Estudiante \#35 del grado 602, 2019), "es buena persona y hace buen trabajo" (Estudiante \#35 del grado 602, 2019), "hacemos un buen equipo, me hace reír" (Estudiante \#16 del grado 603, 2019), "es parte de mis mejores amigos y la paso mejor que con otros" (Estudiante \#11

del grado 603, 2019). Los estudiantes con discapacidad cognitiva y sus compañeros muestran empatía y aceptación mutua que permite la inclusión de estos dentro de los grupos de estudiantes en el curso. En esta medida, Berger y Luckmann refieren que las interacciones conllevan a una carga afectiva y fomentan la adhesión emocional que posteriormente permite la socialización primaria entre actores, en este sentido, los estudiantes del grado sexto se identifican con los otros significantes en una variedad de formas emocionales manifestadas por los mismos en elementos que causan agrado como la amistad, actitudes propias y componentes académicos, esa identificación proporciona en los estudiantes de grado sexto una identificación propia, "el individuo llega a ser lo que los otros significantes lo consideran” (Berger y Luckmann, 1986, p. 167), en esta línea los estudiantes con 
discapacidad cognitiva se convierten en personas divertidas, colaborativas, especiales, amigas y comprometidas académicamente para sus pares académicos.

\section{Discusión y Conclusión}

Como conclusión general y entre los elementos clave para haber realizado el proceso de análisis fue comprender el significado que denotan los estudiantes del grado sexto a la discapacidad y discapacidad cognitiva, en vista de que por medio de las valoraciones que se le brindan estos términos se establecen interacciones entre los actores partícipes de una realidad social, por consiguiente en las narraciones de los sujetos se evidencia el vestigio de déficit, refieren a expresiones como limitaciones, deficiencias, capacidades y funcionamiento, elementos discursivos que reflejan la alteridad y denotan a los estudiantes con discapacidad cognitiva los atributos sociales de raro o lento, que a su vez propicia formas de interacción como la condescendencia que permite ver al otro como un sujeto subordinado, con características diferentes a lo "común", propiciando procesos de distanciamiento social enmarcados en el tipo de interacción de indiferencia, o procesos de agresión directa enmarcados en interacciones de hostilidad, conllevando a que los estudiantes con discapacidad cognitiva ocupen los lugares periféricos, encontrándose en los posicionamientos de rechazado, promedio e ignorado. Sin embargo, no hay que desconocer, que en algunas interacciones los estudiantes identifican a sus compañeros con discapacidad cognitiva como pares e iguales denotando valoraciones que permiten entenderlos como unos sujetos comprometidos en sus actividades académicas, personas amigables y especiales marcando la adhesión emocional a este tipo de interacción. En coherencia, hay que mencionar que las interacciones sociales competen a la inclusión educativa de los estudiantes, a lugares sociales, en este caso a espacios académicos que permiten la socialización. Sin embargo, muchas de las interacciones ya mencionadas aluden a formas de discriminación, etiquetaje y exclusión dentro de las mismas aulas de clase, que desfavorecen el aprendizaje social y el proceso de socialización de las PCD.

Por otra parte, el proceso inclusivo de los estudiantes con discapacidad cognitiva es un ejercicio diario, que se ha visto permeado por las interacciones entre los diferentes actores estudiantiles dentro del contexto regular, propiciado como espacio para la construcción de nuevas realidades que estimulan o no la socialización primaria. Sin embargo, la implantación de estas prácticas inclusivas exige transformaciones en las estructuras sociales, no obstante, al interior de la Institución Educativa Villemar El Carmen se da mayor peso a los aspectos pedagógicos, restándole relevancia al aspecto social que remiten de manera indirecta a prácticas de exclusiónincluyente.

En último, la presente investigación pretende contribuir en el campo de la discapacidad de las ciencias sociales desde la visión social, y al Trabajo Social, mediante una perspectiva ligada a lo humano enmarcandose en los modelos de convergencia, que rompen con los marcos tradicionales de la profesión, dirigidos al asistencialismo y centrados en la individualidad del sujeto desde un modelo médico rehabilitador, originando lecturas dentro de la profesión que apuntan a estudios relacionados con capacidades, habilidades, rehabilitación e integración. Por lo cual, el presente trabajo se encaminó hacia la mirada construccionista que apoya en la creación de nuevos enfoques permitiendo visualizar a la discapacidad como una construcción social. Además, aporta en la generación de conocimiento que posibilita comprender cómo los sujetos enuncian y constituyen el mundo en él que viven considerando sus interacciones y sus experiencias

\section{Referencias bibliográficas}

Álvarez, R.., \& Cázares, C. (2018). Aspectos psicosociales en el proceso de inclusión educativa durante la infancia intermedia. Infancias imágenes,17(1), 9-24. Recuperado de: https://revistas.udistrital.edu.co/index.php/infancias/issue/view/883/290

Arias, L. (2009). Las interacciones sociales que se desarrollan en los salones de clase y su relación con la práctica pedagógica que realiza el docente en el aula. Posgrado y Sociedad, 9(2), 32-57. https://dialnet.unirioja.es/descarga/articulo/3662261.pdf

Bautista, M., Turnbull, B., Saad, E. y Vidal, A., (2016). La interacción de alumnos con discapacidad y sus pares en la escuela regular. Psicología Iberoamericana [en línea] 2016, 24 (Julio-diciembre). Recuperado de: https://www.redalyc.org/jatsRepo/1339/133947583003/html/index.html 
Benito, J. (2010). Educación y exclusión social. Revista Interuniversitaria de Formación del Profesorado, 24(3), 17- 24.

Berger P. y Luckman T. (1968) La construcción social de la realidad. Amorrourtu editores. Recuperado de: https://zoonpolitikonmx.files.wordpress.com/2014/09/la-construccic3b3n-social-de-la-realidad-berger- luckmann.pdf

Berger, P. L., Luckmann, T., \& Estruch, J. (1997). Modernidad, pluralismo y crisis de sentido. Barcelona: Paidós. Recuperado de: http://pdfhumanidades.com/sites/default/files/apuntes/Berger\%20\%26\%20Luckmann\%20-

\%20Modernidad\%2C\%2opluralismo\%20y\%2ocrisis\%20de\%2osentido.pdf

Coie, J. D., Dodge, K. A. y Kupersmidt, J. B. (1990). Peer group behavior and social status. En S. R. Asher y J. D.Coie (Eds.), Peer rejection in childhood (pp. 17-59) New York: Cambridge University Press.

Congreso de Colombia (2013). Ley estatutaria 1618 del 27 de febrero de 2013. Recuperado de: http://wsp.presidencia.gov.co/Normativa/Leyes/Documents/2013/LEY\%201618\%20DEL\%2027\%20DE\%20FE BRERO\%20DE\%202013.pdf

Darling-Hammond, L. (2001). El derecho de aprender. Crear buenas escuelas para todos. (trad. de F. Marhuenda y A. Portela) Barcelona: Ariel [ V.O.: The right to learn. San Francisco: Jossey Bass,1997].

Díaz-Jiménez, R. M. (2011). Trabajo social y discapacidad intelectual en centros residenciales y de día en Andalucía. Una aproximación desde la teoría fundamentada (Doctoral dissertation, Universidad Pablo de Olavide). Donoso, T. (2004). Construccionismo Social: Aplicación del Grupo de Discusión en Praxis de Equipo Reflexivo en la Investigación Científica. Revista de Psicología de la Universidad de Chile. Vol. XIII, No 1: Pág. 9-20. 2004.

https://core.ac.uk/download/pdf/46532554.pdf

Echeita, G. y Simón, C. (2007). La contribución de la educación escolar a la calidad de vida de las personas con discapacidad. Ante el desafío de su inclusión social. Recuperado de: http://www.repositoriocdpd.net:8080/bitstream/handle/123456789/668/CL_EcheitaG_ContribucionEdu cacionCalidad_2007.pdf?sequence $=1$

Estévez López, E., Martínez Ferrer, B., \& Jiménez Gutiérrez, T. I. (2009). Las relaciones sociales en la escuela: el problema del rechazo escolar. Educational Psychology, 15(1), 45-60. Recuperado de: https://journals.copmadrid.org/psed/archivos/ed2009v15n1a6.pdf

Gergen, K. 1996. Realidades y relaciones. Aproximaciones a la construcción social. Barcelona: Paidós.

Gifford-Smith, M. E. y Brownell, C. A. (2003). Childhood Peer Relationships: Social Acceptance, Friendships, and Social Network. Journal of School Psychology, 41 (4), 235-284.

Hernández, R (2014) Metodología de la investigación. Sexta Edición. Mc. Graw Hill Education. Recuperado de: http://observatorio.epacartagena.gov.co/wp-content/uploads/2017/08/metodologia-de-la-investigacion-_ sextaedicion.compressed.pdf

Hill, D. K. y Merrell, K. W. (2009). Characteristics of "controversial" children: an exploration of teacher and parent

social behavior rating scale data sets. Psychology in the Schools, 41(5), 497- 507.

ICBF, (2010). Orientaciones pedagógicas para la atención y la promoción de la inclusión de niñas y niños menores de seis años con Discapacidad Cognitiva. Cap I: Lo que debemos saber sobre la discapacidad cognitiva. Recuperado de: https://www.icbf.gov.co/sites/default/files/cartilla-cognitiva-7.pdf

López, P. (2013) Realidades, Construcciones y Dilemas. Una revisión filosófica al construccionismo social. Cinta moebio 46: 9-25. https://scielo.conicyt.cl/pdf/cmoebio/n46/arto2.pdf

Newcomb, A. F., Bukowski, W. M. y Pattee, L. (1993). Children's peer relations: a meta-analytic review of popular, rejected, neglected, controversial, and average sociometric status. Psychological Bulletin, 113 (1), 99-128.

OMS (2001). Clasificación Internacional del Funcionamiento, la Discapacidad y la Salud. Recuperado de: https://apps.who.int/iris/bitstream/handle/10665/43360/9241545445_spa.pdf;jsessionid=A1CA2D1A5E30 E3B7FOCA36CDD56E5E32? sequence $=1$

Osler, A. y Starkey, H. (2005). Changing Citizenship. Democracy and Inclusion in Education. Nueva York: Open University Press.

Perdomo, M. (2002). Socioconstruccionismo y cultura: relaciones, lenguaje y construcción cultural. Recuperado de: https://repository.icesi.edu.co/biblioteca digital/bitstream/item/3767/1/Socioconstruccionismo cultura 2002.pdf

Puga González, M. D., \& Abellán, A. (2004). El proceso de discapacidad: un análisis de la encuesta sobre discapacidades. Deficiencias y estado de salud. Recuperado de: http://envejecimiento.csic.es/documentos/documentos/puga-discapacidad-01.pdf

Ramírez Î́niguez, A. A. (2016). Repensar la inclusión social desde la educación: algunas experiencias en América Latina. Revista Internacional de Educación para la Justicia Social (RIEJS).

Rincón, A. (2013). Aportes para estudiantes, docentes y familias del proceso de inclusión de niños, niñas, jóvenes en condición de discapacidad en el contexto escolar regular, una mirada desde el construccionismo social. Fundación Centro Internacional De Educación y Desarrollo Humano CINDE, Convenio Universidad Pedagógica Nacional.

https://repository.cinde.org.co/bitstream/handle/20.500.11907/1467/RinconVega2013.pdf?sequence=1\&i sAllowed=y

Ritacco, M. y Luengo, J. (2012). Las políticas de los centros educativos y las buenas prácticas en contextos de exclusión social y educación en la Comunidad Autónoma de Andalucía. Revista Qurriculum, 25, 151-170.

Rosato, A., Alfonsino, A., Almeida , M., Angelino, C., Kippen, E., Sánchez, C. y Priolo, M. (2009). El papel de la ideología de la normalidad en la producción de discapacidad. Ciencia, Docencia y Tecnología, vol. XX, núm. 39, noviembre, 2009. Universidad Nacional de Entre Ríos, 87-105. https://www.redalyc.org/pdf/145/14512426004.pdf

Salazar, C. (2009). Integración Escolar: El puente Entre la Segregación y la Inclusión.

Salinas, M. (2014). Actitudes de estudiantes sin discapacidad hacia la inclusión de estudiantes con discapacidad en la educación superior. (Doctoral dissertation, Universitat Autònoma de Barcelona). Recuperado de: https://www.tdx.cat/handle/10803/284953\#page=196

Soto, N. (2007). La atención educativa de niños, niñas y jóvenes considerados con necesidades educativas especiales: una mirada desde la integración y desde la inclusión (Tesis de doctorado en Ciencias Sociales). Universidad de ManizalesCinde, Manizales, Colombia. Recuperado de: https://repository.cinde.org.co/bitstream/handle/20.500.11907/470/SotoBuilesNorelly2007.pdf?sequenc e=1\&isAllowed $=y$

Tünnermann, C. (2011). El constructivismo y el aprendizaje de los estudiantes. Universidades, 61(48), 21-32. Recuperado de: http://www.redalyc.org/pdf/373/37319199005.pdf 